\title{
Avaliação da alteração dimensional linear de hidrocoloides irreversíveis através do método fotográfico
}

\author{
Evaluation of linear dimensional change of irreversible hydrocolloids \\ through the photographic method \\ Evaluación de la alteración dimensional linear de los hidrocoloides irreversibles \\ a través del método fotográfico
}

\author{
Humberto GENNARI FILHO ${ }^{1}$ \\ Luciana Mara Negrão ALVES ${ }^{2}$ \\ José Vitor Quineli MAZARO ${ }^{1}$ \\ Paulo Renato Junqueira ZUIM ${ }^{1}$ \\ Karina Helga Leal TURCIO ${ }^{1}$ \\ Aimée Maria GUIOTTI ${ }^{\mathbf{1}}$
}

\author{
${ }^{\text {I}}$ Professores do Departamento de Materiais Odontológicos e Prótese da Faculdade de Odontologia de Araçatuba, \\ Universidade Estadual Paulista, UNESP, 16015-050 Araçatuba - SP, Brasil \\ ${ }_{2}^{2}$ Doutora pela Faculdade de Odontologia de Ribeirão Preto, Universidade de São Paulo, USP \\ 14040-904 Ribeirão Preto - SP, Brasil
}

\begin{abstract}
Resumo
O objetivo deste trabalho foi analisar através de método fotográfico, as alterações dimensionais lineares de três marcas de alginato, dois com tempo de presa estendido (Cavex Color Change e Hidrogum 5) e um convencional (Jeltrate dustless), em quatro períodos de tempo (0, 8, 32 e 80 horas). Material e Método: Com base em um modelo superior de uma boca dentada foi obtido um modelo em resina acrílica, que serviu como padrão para a obtenção dos moldes estudados. Sobre os dentes $14,17,24$ e 27 foram inseridas marcas que serviram como referência de mensuração. Com uma moldeira em resina acrílica confeccionada sobre este modelo, produziram-se cinco moldes para cada marca de alginato, um de cada vez, sendo os mesmos fotografados em estativa e máquina fotográfica Nikon D50, com distância preestabelecida para todas as fotos. As imagens foram mensuradas no programa Corel Draw. Com base nos valores obtidos dos moldes, e do modelo padrão foi possível estabelecer as alterações dimensionais lineares que ocorreram nas três marcas de alginato, nos tempos estabelecidos. Resultados: Não houve diferença estatística significante entre as três marcas de alginato estudadas. Conclusão: Concluiu-se que a estabilidade dimensional linear dos moldes dos três alginatos estudados estão inseridos num nível aceitável para produzir modelos adequados e que não houve diferença estatística significante entre eles.

Descritores: Alginatos; Materiais para Moldagem Odontológica; Precisão da Medição Dimensional.
\end{abstract}

\begin{abstract}
The objective of this study was to analyze linear dimensional changes of three brands of alginate, two with extended setting time (Cavex Color Change and Hidrogum 5) and a conventional (Jeltrate dustless) in four periods (0, 8, 32 and 80 hours) through photographic method. Material and methods: A model in acrylic resin, which served as the standard for obtaining the molds studied was obtained from a superior dentate mouth. Tags were entered on teeth 14, 17, 24 and 27 and it served as a measurement reference. A acrylic resin tray was used to produce five molds for each alginate, one at a time, photographed in the stand and Nikon D50 camera with predetermined distance for all pictures. The images were measured in Corel Draw program. Based on the values obtained from the molds, and the standard model was possible to establish the linear dimensional changes that have occurred in the three brands of alginate, at the appointed times. Results: There was no significant statistical difference between the three alginate brands studied. Conclusion: It was concluded that the linear dimensional stability of the molds of the three studied alginates are inserted at an acceptable level to produce appropriate models and that there was no statistically significant difference between them.
\end{abstract}

Descriptors: Alginates; Dental Impression Materials; Dimensional Measurement Accuracy.

\section{Resumen}

El objetivo de este estudio fue analizar a través del método fotográfico, lãs alteraciones dimensionales lineales de tres marcas de alginato, dos con tiempo de fraguado prolongado (Cavex color change y Hidrogum 5) y un convencional (Jeltrate dustless) en cuatro períodos de tiempo $(0,8,32$ y 80 horas). Métodos: Con un modelo superior de una boca dentada se obtuvo un modelo en resina acrílica, que sirvió como el estándar para la obtención de los moldes estudiados. En los dientes 14, 17, 24 y 27 se colocaron marcas que sirvió como referencia de medición. Con una cubeta de acrílico hecho en este modelo se produció cinco moldes para cada marca de alginato, uno a la vez, y se fotografiaron en el soporte y la cámara Nikon D50 con la distancia predeterminada para todas las imágenes que se midieron en el programa Corel Draw. Com base en los valores obtenidos a partir de los moldes, y el modelo estándar ha sido posible establecer los cambios dimensionales lineares que se han producido em las tres marcas de alginato, en los tiempos señalados. Resultados: No hubo diferencia estadísticamente significativa entre las tres marcas de alginato estudiados. Conclusión: Se concluyó que la estabilidad dimensional linear del molde de los tres alginatos estudiados se insertan en un nivel aceptable para producir modelos apropiados y que no hubo diferencia estadísticamente significativa entre ellos.

Descriptores: Alginatos; Materiales de Impresión Dental; Precisión de la Medición Dimensional.

\section{INTRODUÇÃO}

Os materiais de impressão odontológicos, em particular os hidrocolóides irreversíveis, também conhecidos como alginatos, são os materiais mais comumente usados para gerarem modelos em numerosas aplicações, incluindo plano de tratamento para trabalhos restauradores, ortodônticos e fabricação de próteses. Tal como acontece com qualquer hidrocolóide, os alginatos são aproximadamente $85 \%$ água e propensos a distorção causada pela expansão associada à embebição (absorção de umidade) ou sinérese. Consequentemente, as impressões com alginato não são estáveis dimensionalmente o que conduz a uma diminuição da precisão ao longo do tempo. Portanto, a recomendação é o vazamento imediato, não devendo ultrapassar um período de 30 minutos $^{1}$. 
A estabilidade dimensional é definida como sendo uma habilidade do material para manter sua precisão dimensional ao longo de um determinado período de tempo. Sabemos que a precisão dos modelos de gesso é de grande importância para criar restaurações protéticas de alta qualidade. Muitos materiais de impressão sofrem contração durante sua presa enquanto o gesso expande. Contudo, a precisão dos modelos depende principalmente do momento do vazamento e da compatibilidade do material de impressão com o gesso utilizado. Geralmente, um curto período de tempo produz modelos mais precisos. Por esta razão, o intervalo de tempo envolvido no envio de um molde para o laboratório de prótese dentária dificulta o uso do alginato sem o vazamento quase imediato ${ }^{2}$. Recentemente os fabricantes apresentaram novos produtos para o mercado, com a alegação de que estes novos alginatos são estáveis até 120 horas; e chamados de "extended-pour" (vazamento estendido) ou "alginatos de 100 horas"3.

Assim, algumas pesquisas foram realizadas com o intuito de comprovar estas afirmações e dentre elas, Sedda et $\mathrm{al}^{4}$ que avaliaram a precisão dimensional em modelos obtidos através da moldagem com cinco tipos de alginatos armazenamento imediato, 24, 72 e 120. Os resultados mostraram que após 24 horas de armazenamento, somente o alginoplast e o Hidrogum 5 mostraram-se iguais ao modelo mestre e que após 72 e 120 horas somente o Hidrogum 5 permaneceu dimensionalmente estável. Da mesma forma, Imbery et al. $^{5}$ (2010), conduziram um estudo para determinar a precisão dimensional de dois alginatos com vazamento estendido (Cavex Color Change e Jeltrate Plus Antimicrobial) armazenados durante cinco dias. Concluíram que os modelos gerados imediatamente e após 5 dias, para ambos os materiais, não apresentaram diferenças estatística significantes do modelo padrão, e que os materiais utilizados produzem moldes que podem ser preservados por cinco dias. Erbe et al. $^{6}$ citam que o tempo e as condições de armazenamento dos moldes de alginato afetam a sua precisão e por sua vez a precisão do modelo definitivo. Contudo, as recomendações para o armazenamento adequado são baseadas em pressupostos teóricos ao invés de fatos e que para uma estabilidade ótima as impressões devem ser vazadas o mais rápido possível.

Dorner et al. ${ }^{7}$ avaliaram o efeito da desinfecção com hipoclorito de sódio a $1 \%$ sobre a rugosidade superficial e alteração dimensional de modelos obtidos com quatro alginatos: Jeltrate plus, Hidrogum, Hidrogum 5 e Cavex concluíndo que mesmo após cinco dias não houve diferenças significativas em relação à alteração dimensional entre as marcas havendo um ligeiro aumento na rugosidade daqueles que utilizaram o hipoclorito.

Considerando as diferentes pesquisas que alegam propriedades distintas entre os hidrocolóides quanto aos seus tempos de vazamento, o objetivo desta pesquisa foi comparar as alterações dimensionais de três marcas de alginatos, dois com tempo estendido e um com tempo regular, analisados nos tempos de $0,8,32$ e 80 horas através de um método fotográfico associado à leitura por programa de computação. A hipótese formulada ao presente trabalho é de que os alginatos com tempo estendido apresentem alteração dimensional linear inferior ao alginato convencional.

\section{MATERIAL E MÉTODO}

O padrão para a obtenção dos moldes estudados foi um modelo de resina acrílica, confeccionado a partir de um modelo de arco maxilar dentado. Os dentes 14, 17, 24 e 27 tiveram as superfícies oclusais aplainadas, e complementadas com resina acrílica Duralay (Polidental, Cotia, SP, Brasil), e sobre esta, traçado um sinal "+" com disco 7/8 (Horico, Wilcos, Germany) sendo a intersecção destes traços a representação dos pontos que foram mensurados (Figura 1), constituindo dois segmentos: A e B.

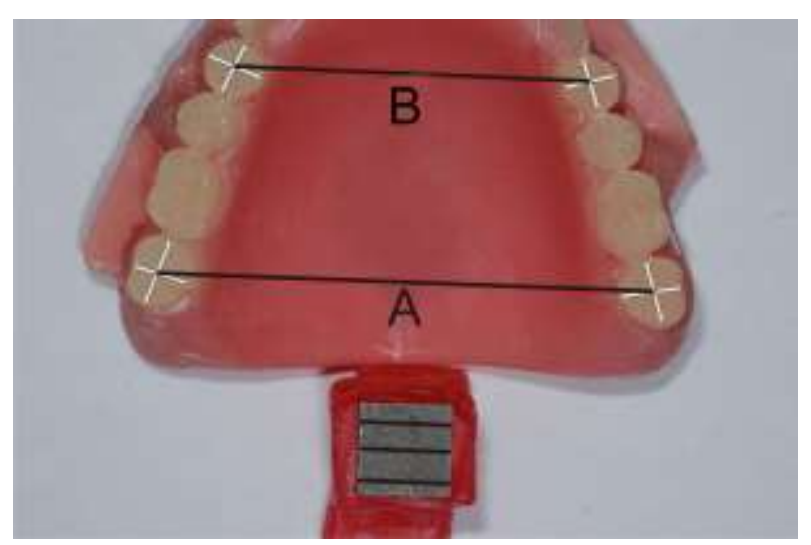

Figura 1: Modelo Padrão em acrílico, referência para obtenção dos moldes.

Sobre este modelo aliviado com cera rosa $\mathrm{n}^{\circ} 7$ (Polidental Ind. e Com. Ltda. Cotia, Brasil), com espessura de 3,75 mm (três lâminas de cera), foi construída uma moldeira em resina acrílica rosa e perfurada em toda a sua extensão para permitir o extravasamento do material de moldagem e ao mesmo tempo possibilitar sua retenção à mesma. Para a obtenção de moldes com a mesma espessura, o modelo em resina acrílica apresentava no seu contorno, uma extensão para frear o aprofundamento da moldeira e ao mesmo tempo, um parafuso adaptado para facilitar sua remoção (Figura 2).

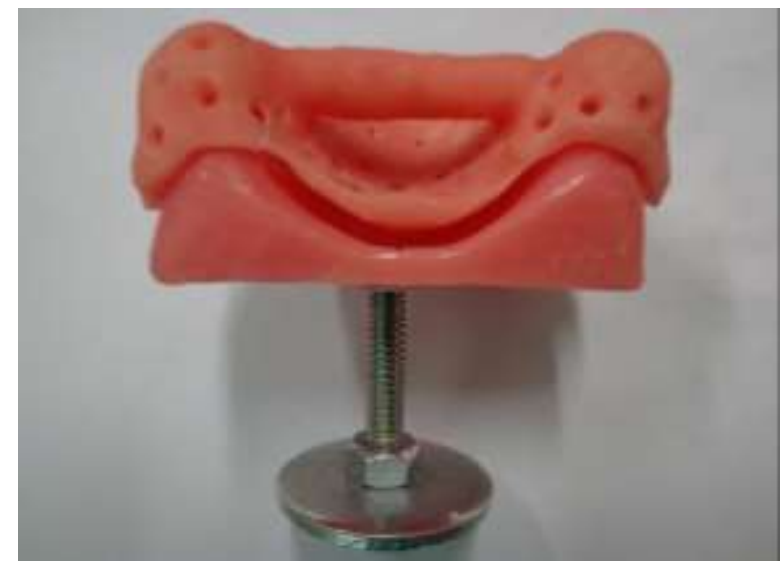

Figura 2: Modelo e Moldeira adaptada para obtenção dos moldes.

Os alginatos utilizados foram: Cavex Color Change (Cavex Holland BV Haarlem, Netherland), Hidrogum 5 (Zhermack, Badia Polesine, Italy) e Jeltrate Dustless (Dentsply Indústria e Comércio Ltda, Petrópolis, Brasil), que constituíram três grupos:

1. CCC - representando o alginato Cavex Color Change

2. H5 - representando o alginato Hidrogum 5

3. JD - representando o alginato Jeltrate Dustless

Os materiais foram proporcionados, espatulados e carregados nas moldeiras de acordo com as especificações de cada fabricante, para a obtenção de 5 moldes de cada material, um de cada vez, perfazendo um total de 15 moldes, mantidos em sacos plásticos herméticamente fechados, secos, em temperatura ambiente e analisados da seguinte maneira:

a) Fotografia: os moldes foram posicionados na base de uma estativa (Asahi Pentax, Japan) acoplada a uma máquina Nikon D50, com lente macro e flash circular da marca Sigma, com a distância câmera/objeto determinada e mantida igual para todas as fotografias, realizadas com alta resolução. Juntamente com o molde foi posicionado um bloco metálico com dimensões de 10 x $10 \mathrm{~mm}$ para 
determinar a verdadeira grandeza da fotografia possibilitando dimensionar os valores obtidos, em valores reais. Os moldes foram fotografados nos tempos de $0 \mathrm{hs}$ (imediatamente após a moldagem), 8, 32 e 80 horas após a moldagem (Figura 3).

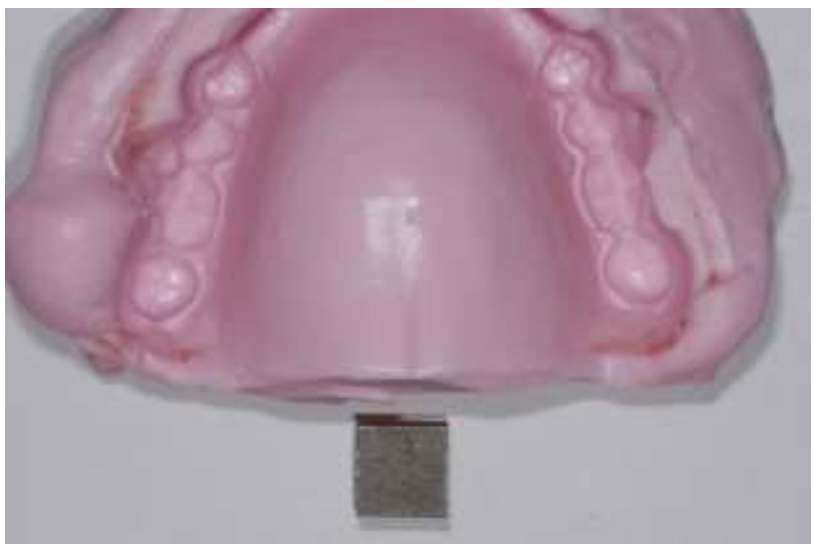

Figura 3: Fotografia do molde com bloco metálico.

b) Mensuração: O programa para mensuração das distâncias entre os pontos determinados foi o Corel Draw X3 Graphics Suite (Corel Corporation - USA). As fotografias foram abertas no programa e pela ativação do recurso "caneta", foram traçadas linhas correspondentes ao sinal "+"de cada dente, para determinar o momento da intersecção e definir o ponto de mensuração nos quatro dentes (Figura 4).

A seguir, com o mesmo recurso, as distâncias entre os dois molares e os dois pré-molares foram medidas pelo próprio programa, por três vezes para cada fotografia, determinando uma média aos valores dos segmentos A e B sendo a mesma técnica aplicada ao bloco metálico (Figura 5).

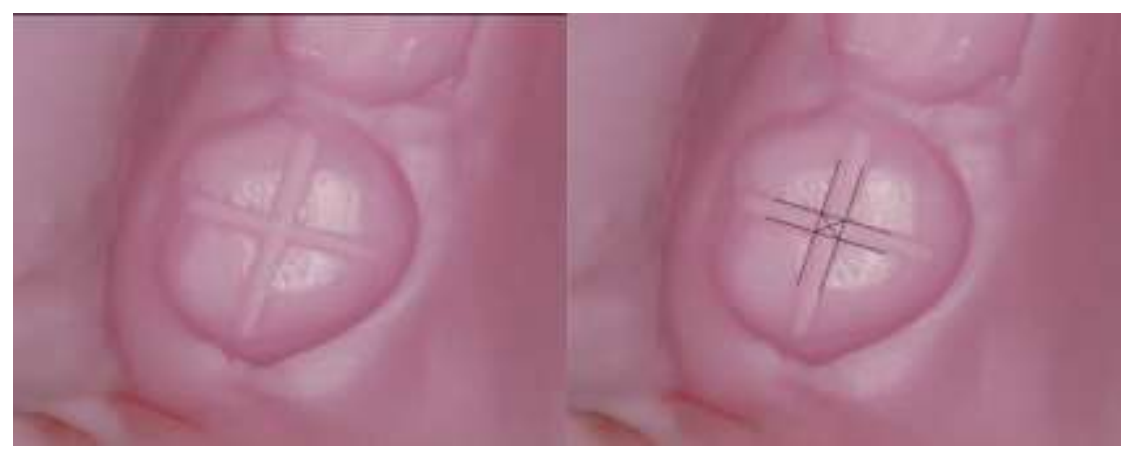

Figura 4: Fotografia com os traçados determinando o ponto de intersecção.

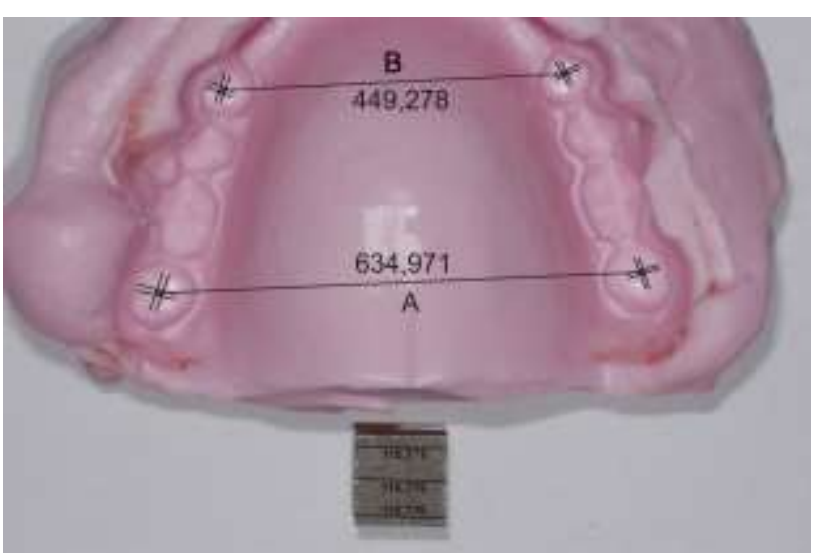

Figura 5: Fotografia com a mensuração entre os pontos determinados.

Para a obtenção dos valores reais, os mesmos foram transformados com base no bloco metálico que media $1 \mathrm{~cm} \mathrm{x}$ $1 \mathrm{~cm}$ (valor de referência) por uma regra de três simples considerando como exemplo:

Mensuração horizontal do bloco $=118,776 \mathrm{~mm}$

Mensuração entre os molares $=634,971 \mathrm{~mm}$

Mensuração entre os pré-molares $=449,278 \mathrm{~mm}$

Assim temos:

$118,776 \mathrm{~mm}=10 \mathrm{~mm}$

$634,971 \mathrm{~mm}=\mathrm{X}$

$\mathrm{X}=53,459 \mathrm{~mm}$ ou $5,345 \mathrm{~cm}$ (Valor real entre os molares).
Para a Análise Estatística, foi realizado o Teste de Normalidade dos Dados, apontando uma Distribuição Normal, de forma que se pôde empregar um Teste Paramétrico. Assim, aplicou-se a Análise de Variância (ANOVA) para 02 Fatores de Variação (Marca de Alginato e Tempo).

\section{RESULTADOS}

As médias dos segmentos A e B do Modelo Padrão e dos alginatos testados estão contidos nas tabelas 1 e 2 , convertidos em centímetros.

Tabela 1. Médias em cm do segmento " $A$ " para o modelo padrão e para as 05 repetições dos alginatos Cavex, Jeltrate e Hidrogum 5, nos diferentes períodos de armazenagem

\begin{tabular}{ccccc}
\hline \multicolumn{5}{c}{ Segmento A } \\
\hline Períodos & Modelo & Cavex & Jeltrate & Hidrogum \\
0 hs & 5,399 & 5,353 & 5,347 & 5,351 \\
8 hs & 5,399 & 5,345 & 5,348 & 5,351 \\
32 hs & 5,399 & 5,339 & 5,354 & 5,358 \\
80 hs & 5,399 & 5,346 & 5,355 & 5,35 \\
\hline
\end{tabular}

Tabela 2. Médias em cm do segmentos "B" para o modelo padrão e para as 05 repetições dos alginatos Cavex, Jeltrate e Hidrogum 5, nos diferentes períodos de armazenagem

\begin{tabular}{ccccc}
\hline \multicolumn{5}{c}{ Segmento B } \\
\hline Períodos & Modelo & Cavex & Jeltrate & Hidrogum \\
$0 \mathrm{hs}$ & 3,851 & 3,8 & 3,79 & 3,799 \\
$8 \mathrm{hs}$ & 3,851 & 3,793 & 3,788 & 3,797 \\
$32 \mathrm{hs}$ & 3,851 & 3,793 & 3,794 & 3,8 \\
$80 \mathrm{hs}$ & 3,851 & 3,787 & 3,793 & 3,793 \\
\hline
\end{tabular}

As tabelas 3 e 4 mostram as diferenças entre as médias do Modelo Padrão e os materiais testados para os segmentos A e B, nos diferentes períodos de armazenagem.

Tabela 3. Diferença em $\mathrm{cm}$ entre as médias do modelo padrão e os materiais testados, nos diferentes períodos de armazenagem para o segmento "A".

\begin{tabular}{cccc}
\hline Períodos & Cavex & Jeltrate & Hidrogum \\
$0 \mathrm{hs}$ & 0,046 & 0,052 & 0,048 \\
$8 \mathrm{hs}$ & 0,054 & 0,051 & 0,048 \\
$32 \mathrm{hs}$ & 0,060 & 0,045 & 0,041 \\
$80 \mathrm{hs}$ & 0,053 & 0,044 & 0,049 \\
\hline
\end{tabular}

Tabela 4. Diferença em cm entre as médias do modelo padrão e os materiais testados, nos diferentes períodos de armazenagem para o segmento "B".

\begin{tabular}{cccc}
\hline Periodos & Cavex & Jeltrate & Hidrogum \\
$0 \mathrm{hs}$ & 0,051 & 0,061 & 0,052 \\
$8 \mathrm{hs}$ & 0,058 & 0,063 & 0,054 \\
$32 \mathrm{hs}$ & 0,058 & 0,057 & 0,051 \\
$80 \mathrm{hs}$ & 0,064 & 0,058 & 0,058 \\
\hline
\end{tabular}

$\mathrm{Na}$ análise estatística os valores apresentados para o cálculo, referem-se às diferenças de cada medida dos corpos de prova para o Modelo Padrão, porque foi o intuito comparar as diferenças entre alginatos e momentos de vazamento, em relação ao Modelo Padrão. Assim, para o Segmento "A" não houve diferenças estatísticas significantes entre os alginatos e os momentos (Alginatos $\mathrm{p}=$ 0,07; Tempos $\mathrm{p}=0,12$ ) e, para o Segmento "B", não houve diferenças estatísticas significantes entre os alginatos e os momentos (Alginatos: $\mathrm{p}=0,070$; tempos $\mathrm{p}=0,22$ ).

\section{DISCUSSÃO}

Próteses bem sucedidas requerem a aplicação sensata de experiência e conhecimento dos materiais odontológicos, pois um modelo preciso é um passo importante para o sucesso de vários tratamentos dentários. A demora em vazar o molde é uma das razões que provocam alterações, ameaçando a exatidão dimensional da prótese. Os materiais de moldagem devem ter capacidade de boa reprodução de 
detalhes e fidelidade e devem ser dimensionalmente estáveis ao longo do tempo. No alginato a água pode estar livre ou ligada e devido à sua evaporação ou embebição o material fica suscetível ao aumento volumétrico ou diminuição. A perda de água depende da diminuição da entropia, da complexa pressão osmótica, das mudanças na energia livre de Gibbs, da cinética de difusão das mudanças de gradiente existentes entre o sol-gel e dos componentes do ambiente ${ }^{8}$. Fernandes et $\mathrm{al}^{9}$ 2014, citam que a estabilidade dimensional dos alginatos podem estar relacionados às suas diferentes composições químicas. No entanto, pouca informação é disponibilizada pelos fabricantes e não há literatura recente que explique a influência dos componentes químicos individuais na estabilidade dimensional desses materiais $\mathrm{e}$ que esta, provavelmente esteja relacionada à habilidade do material em manter a água no interior de sua massa, do que à quantidade global de água presente em cada molde. Por outro lado, Fonte Boa et $\mathrm{al}^{10} 2016$, acreditam que a relação cálcio/sódio, carga/polímero, peso molecular dos polímeros de ácido algínico, além de outros constituintes específicos, são fatores relacionados às alterações dimensionais. Estudos de ressonância magnética nuclear mostram que alginatos com alta razão cálcio/sódio perdem água mais rapidamente do que aqueles com baixa razão cálcio/sódio e, quando há polímeros de ácido algínico com baixo peso molecular, a estabilidade dimensional é melhorada ${ }^{11}$. Os alginatos cromáticos são alcalinos durante a mistura inicial, mas quando geleificam diminuem o $\mathrm{pH}$ próximo à neutralidade ${ }^{12}$ além dos aditivos que podem influenciar a estabilidade dimensional, mas têm um papel benéfico.

A literatura pertinente ao estudo dos hidrocolóides mostra certa incoerência em algumas comparações uma vez que alguns autores ${ }^{4,5}$, com base em suas pesquisas, atestam a versão dos fabricantes quanto à alteração dimensional, enquanto outros contradizem. Pode ser observado pelos trabalhos de pesquisadores ${ }^{13,14,15,16}$ que os novos alginatos sofrem alterações antes do tempo previsto pelos fabricantes. Devemos considerar ainda que todas as mensurações dos trabalhos referidos foram realizadas em modelos de gesso, obtidos de suas respectivas impressões, e que estes sofrem expansão, portanto com medidas não coincidentes com as dos moldes. Seria interessante que estas mensurações pudessem ser realizadas diretamente nos moldes eliminando assim uma variável que contribui para a apreciação da verdadeira alteração dimensional.

Assim, no presente trabalho, devemos considerar que a análise dos valores das médias dos alginatos estudados, confrontados com os valores do modelo Padrão, mostra diferenças numéricas que podem ser entendidas como alteração dimensional linear que apesar de serem mínimas, são reais. Kulkarni \& Thombare $^{8}$ (2015) citam que a estabilidade dimensional do material de impressão em relação ao modelo padrão mostrou a maior discrepância em torno de 0,127 $\mathrm{mm}$ e aqueles com tempo estendido foi de $0,076 \mathrm{~mm}$, no vazamento do molde. No presente trabalho, no segmento posterior (A) foi encontrada uma variação de 0,60 $\mathrm{mm}$ a $0,41 \mathrm{~mm}$ e no segmento anterior (B) houve uma variação de $0,63 \mathrm{~mm}$ a $0,51 \mathrm{~mm}$ sem considerar o tipo de material, se com tempo estendido ou não. De acordo com Imbery et al. ${ }^{5}$ (2010) e Rohanian et al. ${ }^{17}$ (2016) um modelo de gesso para ser clinicamente aceitável não deve apresentar valores maiores do que $75 \mu \mathrm{m}$ de discrepância do que se pretende reproduzir. Os autores consideram aceitável um percentual em torno de $0,5 \%$ de alteração dimensional para os alginatos. No presente trabalho, em todos os materiais estudados, os valores de contração foram maiores, sendo o menor encontrado $(0,7 \%)$ no hidrogum 5 , no segmento "A", após 32 horas de armazenagem.

Durante a geleificação, por causa do esforço de pressão desigual, pode ocorrer um stress que é relaxado após a remoção da moldeira da boca, resultando em uma impressão distorcida ${ }^{4}$. Os estudos de $\operatorname{Sedda}^{4}$ (2008) e Frey et al. ${ }^{18}$ (2005) mostraram uma distorção que varia entre 100 a 500 micrometros durante a moldagem.

Fernandes et al. ${ }^{9}$ (2014) mencionam que os alginatos de última geração quando armazenados de forma conveniente podem ser considerados estáveis para obtenção de modelos de estudo, placas interoclusais e de estruturas para próteses parciais e que sua alteração é equivalente à silicona de adição Express, mas que em seu estudo o Cavex color change sofreu a maior alteração dimensional linear, estatisticamente diferente dos demais materiais estudados ou seja, Hidrogum 5 e Jeltrate. Embora nos nossos resultados os materiais estudados não mostraram diferenças estatísticas, existe semelhança, pois o material Cavex apresentou as maiores alterações nos tempos de 32 e 80 horas e o Hidrogum 5 e o Jeltrate mostraram-se bastante similares nos tempos de 32 e 80 horas, apresentando as menores alterações lineares. No entanto, ainda que seja interessante produzir modelos de gesso a partir de impressões de alginato o mais rápido possível após a remoção do molde da boca, o pressuposto de que moldes em alginato jamais podem ser armazenados em condições satisfatórias, para só serem vazados mais tarde, não é absolutamente um dogma imutável em relação aos materiais de última geração ${ }^{17}$.

Devemos destacar que os alginatos se manipulados convenientemente, seguindo as orientações do fabricante, utilizando moldeiras apropriadas e promovendo um acondicionamento hermético, dentro do tempo estudado, produzirão modelos apropriados para a execução de vários tipos de trabalhos protéticos.

\section{CONCLUSÃO}

1. A estabilidade dimensional linear dos moldes dos três alginatos estudados esta inserida num nível aceitável para produzir modelos adequados.

2. Não houve diferença estatística significante entre as diferenças apresentadas em relação as 03 marcas de alginatos e momentos de vazamento estudados, referentes a um modelo padrão.

Não foi confirmada a hipótese apresentada considerando que os três alginatos estudados não mostraram diferença estatística significante.

\section{REFERÊNCIAS}

1. Walker MP, Burckhardb J, Mittsc DA, and Williamsd KB. Dimensional change over time of extended-storage alginate impression materials. Angle Orthod. 2010; 80(6):1110-5.

2. Woortman R, Werner R, Gee AJ, Feilzer AJ. Evaluation of the Dimensional Stability of some Impression Materials. Cavex Holland B.V., ACTA, Department of Dental Materials Science, NL. 2003.

3. Todd JA, Oesterle LJ, Newman SM, Shellhart WC Dimensional changes of extended pour alginate impression materials. Am J Orthod Dentofacial Orthop.2013; 143(4 Suppl):S55-63.

4. Sedda M, Casarotto A, Raustia A, Borracchini A. Effect of storage time on the accuracy of casts made from different irreversible hydrocolloids. J Contemp Dent Pract. 2008; 9(4):59-66. 
5. Imbery TA, Nehring J, Janus C, Peter C. Moon PC. Accuracy and dimensional stability of extended-pour and conventional alginate impression materials. J Am Dent Assoc. 2010; 141(1):32-9.

6. Erbe C, Ruf S, Wostmann B, Balkenhol M. Dimensional stability of contemporary irreversible hydrocolloids: Humidor versus wet tissue storage. J Prosthet Dent. 2012; 108(2):114-22.

7. Dorner AR, da Silva JMF, Uemura ES, Borges ALS, Vilas Boas FJ, Yamamoto ETC. Effect of disinfection of irreversible hydrocolloid impression materials with $1 \%$ sodium hypochlorite on surface roughness and dimensional accuracy of dental stone casts. Eur J Gen Dent. 2014; 3(2):113-9.

8. Kulkarni MM, Thombare RU. Dimensional changes of alginate dental impression materials - an in vitro study. J Clin Diagn Res. 2015; 9(8):98-102.

9. Fernandes SL, Franciscone PAS, Manfredi GGP, Cavenago BC, Costa AU, Alcade MP, Borges AFS. Estabilidade dimensional linear de alginatos de última geração em função do tempo de armazenagem dos moldes. Innov Implant J, Biomater Esthet. 2014; $8(1): 18-22$.

10. Fonte-Boa JC, Lanza MDS, Peixoto RTRC, Drummond AF, Sousa EL. Análise dimensional de moldes de alginato após armazenagem. Arq Odontol. 2016; 52(2):117-22.

11. Fellows CM, Thomas GA. Determination of bound and unbound water in dental alginate irreversible hydrocolloid by nuclear magnetic resonance spectroscopy. Dent Mat. 2009; 25(4):486-93.

12. Bayindir F, Yanikoglu N, Duymus Z. Thermal and ph changes and dimensional stability in irreversible Hydorcolloid impression material during setting. Dent Mat J. 2002; 21(2):200-9.

13. Samra APB, Pedrini SCL, Anselmo DEA, Urban VM. Linear-Dimensional stability of hidrocolloid after storage and double-pouring. (Poster Session) IADR General Session. Iguaçu Falls, Brasil, june 21, 2012.

14. Rodrigues SB, Augusto CR, Leitune VCB, Samuel SMW, Collares FM. Influence of delayed pouring on irreversible hydrocolloid properties. Braz Oral Res. 2012; 26(5):404-9.

15. Manfredi GGP, Fernandes SL, Francisconi LF, Francisconi PAS. Alteração linear de modelos de gesso pedra em função do uso de diferentes materiais de moldagem elásticos e do tempo de armazenagem dos moldes. $20^{\circ}$ simpósio Internacional de iniciação científica da USP- 22 a 26 outubro de 2012.

16. Wadhwa SS, Mehta R, Duggal N, Vasudeva K. The effect of pouring time on the dimensional accuracy of casts made from different irreversible hydrocolloid impression materials. Contemp Clin Dent. 2013; 4(3):313-18.

17. Rohanian A, Shabestari GO, Zeighami S, Samadi MJ, Shamshiri AR. Effect of storage time of extended-pour and conventional alginate impressions on dimensional accuracy of casts. J Dent. 2014; 11(6):655-64.

18. Frey G, Lu H, Powers J. Effect of mixing methods on mechanical properties of alginate impression materials. J Prosthodont. 2005; 14(4):221-5.

\section{CONFLITO DE INTERESSES}

Os autores declaram não haver conflitos de interesse.

\section{AUTOR PARA CORRESPONDÊNCIA}

\section{Humberto Gennari Filho}

gennari@foa.unesp.br

Submetido em 02/02/2017 Aceito em 20/02/2017 